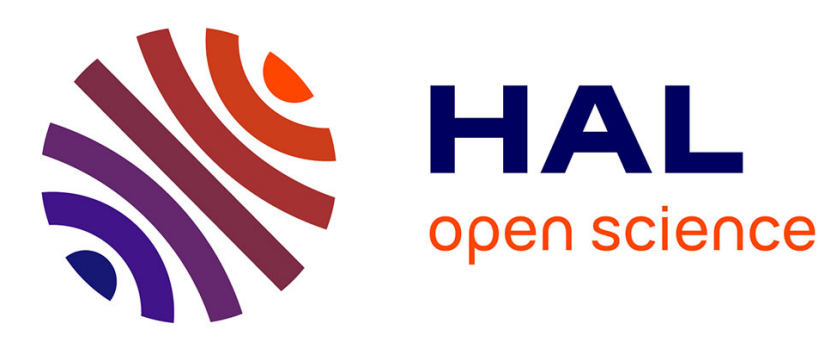

\title{
A Confidence-based Dynamic Optimization Model for Biomedical Image Mosaicking
}

\author{
Romuald Perrot, Pascal Bourdon, David Helbert
}

\section{To cite this version:}

Romuald Perrot, Pascal Bourdon, David Helbert. A Confidence-based Dynamic Optimization Model for Biomedical Image Mosaicking. Journal of the Optical Society of America. A Optics, Image Science, and Vision, 2019, 36 (11), pp.C28. 10.1364/JOSAA.36.000C28 . hal-02297793

\section{HAL Id: hal-02297793 \\ https://hal.science/hal-02297793}

Submitted on 11 Dec 2019

HAL is a multi-disciplinary open access archive for the deposit and dissemination of scientific research documents, whether they are published or not. The documents may come from teaching and research institutions in France or abroad, or from public or private research centers.
L'archive ouverte pluridisciplinaire HAL, est destinée au dépôt et à la diffusion de documents scientifiques de niveau recherche, publiés ou non, émanant des établissements d'enseignement et de recherche français ou étrangers, des laboratoires publics ou privés. 


\title{
A confidence-based dynamic optimization model for biomedical image mosaicking
}

\author{
Romuald Perrot, Pascal Bourdon, David Helbert
}

September 24, 2019

\begin{abstract}
Biomedical image mosaicking is a trending topic. It consists in computing a single large image from multiple observations, and becomes a challenging task when said observations barely overlap or are subject to illumination changes, poor resolution, blur and either highly textured or predominantly homogeneous content. Because such challenges are common in biomedical images, classical keypoint/featurebased methods perform poorly. In this paper, we propose a new framework based on pairwise template matching coupled with a constrained, confidence-driven global optimization strategy to solve the issue of microscopic biomedical image mosaicking. First we provide experimental results that show significant improvement on a subjective level. Then we describe a new validation strategy for objective assessment, which shows improvement as well.
\end{abstract}

Address: XLIM Laboratory, UMR CNRS 7252, University of Poitiers, France, 11 Bd Marie et Pierre Curie, TSA 41123, F-86000 Poitiers, France

I3M, Common Laboratory CNRS-Siemens, University and Hospital of Poitiers, France

Keywords: Image mosaicking, biomedical image processing, image registration, biomedical imaging

\section{Introduction}

Image mosaicking aims at building a large image, sometimes called panoramic image, from a set of subimages pointing to a same visual target. As an example it can be used in microscopic tile scanning or confocal microscopy to increase spatial resolution or solve the issue of small field of view limitations for large biological specimens. This, however, is a challenging task, especially in the context of biomedical imaging where visual targets sometimes barely show decipherable structure, either because (a) subimages have strong gradients but they do not maintain a straight geometry i.e. complex textures (b) large areas are simply missing data and/or displaying homogeneous content. Our work was originally dedicated to multi-photon microendoscopy [1, 2, 3, 4, before we extended it to microscopic tile scanning for the biopsy of transplanted human kidneys and tumor histology.

In this paper, we present a global framework to perform large mosaicking of biomedical images based on a set of scanned images. The used microscopic imaging scanning system acquires hundreds of tiles with a resolution of size $2456 \times 1842$ along a serpentine path.

A full review of mosaicking algorithm is beyond the scope of this paper and we refer the reader to several surveys [5, 6, 7] for an in-depth overview of image mosaicking in the context of biomedical imaging.

Mosaicking is used intensively in astrophysics, where it is used to provide a large-field image from several low-field observations. One key method relies on the Point Response Function (PRF) [8], which provides information on how a sensor responds to a pulse emitter. Intuitively it emulates the response of a given stellar element (e.g. a star). Mosaicking is performed through $1 D$ matching between all responses. Given the fact that stellar elements barely 
move with respect to the acquisition image scale, results are reliable more often than not. However this solution is not applicable in our context as input subimages will not present such strong and immutable features. Mosaicking of low-textured images is however often needed for underwater panoramic reconstructions. Nagaraja et al. 9] proposed to use Local Binary Patterns (LBP) to enhance robustness of the matching process in low textured regions, followed by a classical scheme made of keypoint matching and homography estimation through RANSAC (RANdom SAmple Consensus). This strategy still relies on a keypoint extraction process (here using Difference of Gradient or DoG) similar to [10, thus it is inapplicable with large uniform regions.

Multiview biomedical imaging has received a lot of attention in the last decades. Kölher et al [11] use mosaicking for retinal images. Their method starts with a rough alignment, based on localization of the eye using a tracking method, then precise alignment is computed using intensity based registration. As indicated by the authors, retinal images present large overlap, subjects make small moves, illumination is highly controlled and the number of images is relatively small (10 at most). Zhang et al [12] propose a full 2D framework for mosaicking endoscopic images. They rely on trajectory-pose estimation based on Kalman filters [13] and stereo reconstruction. While this system presents interesting results, it is complex and requires initial camera calibration to work. Miranda-Luna et al. 14] also provide a mosaicking framework for endoscopic image sequences. They rely on Mutual Information [15] measures in order to register pairs of images, then a gradient descentbased optimization framework is employed to correct drifting in long range sequences. Although it handles lens distorsion and loop closure, this approach suffers from requiring large overlaps between images.

The instrumentation in microscopic scanning is constantly evolving and hundreds of images at high pixellic resolution can be acquired with the latest microscopes. The microscopic image stitching has always been subject of many works in microscopic imaging but new contraints are the number, the size of input frames and acquired textures. In 2006, Vercauteren et al presented in [16 a globally consistent alignment of frames with a motion-induced distortion compensation and the non-rigid deformation capture. The global positioning is formalized as an estimation problem on a Lie group and solved by an optimization algorithm. Preibisch et al proposed in [17] a stitching technique from the Fourier-based phase correlation method. The error propagation is reduced by minimizing the sum of all pairwise transfer errors. Loewke et al proposed in [18] a global alignement from deformable surface models after applying a local alignment algorithm. The optimization scheme integrates an intra-image contraints computed on image patches and inter-image constraints modeling the deformation between neighboring images. Bria et al work on a 3D stitching of microscopic images named Terastitcher [19]: on the 2D method, the relative position between each pair of adjacent tiles is found and a globally optimal placement of titles in the mosaic is then computed. The stitching technique proposed by Piccinini et al and named MicroMos in [20] is based on visual information and built the mosaic by incrementally stitching image couples. More recently, Chalfoun et al have proposed a performance-oriented implementation of a stitching technique named $\mathrm{Mi}$ croscopy Image Stitching Tool (MIST) in [21. The translations between adjacent frames are computed from the Fourier-based correlation method. Cumulative errors in the stitched image are then reduced by a global optimization of the computed translations. The mosaic is built from a minimum spanning tree algorithm. A video-mosaicking method has been presented by Kose et al in 22 taking into account the frame deformations, motion artifacts, optical sectioning and resolution between consecutive images.

While part of our application may record such images (multi-photon microendoscopy), the other part (microscopic tile scanning) will not. Moreover, we also need a convenient formalized framework to handle gracefully future works on complex deformations such as odd-shaped optical fiber distortions and not just $2 \mathrm{D}$ or $3 \mathrm{D}$ moves.

Global methods are sometimes employed to deal with loop closure in cases such as panoramic image reconstruction from video sequences. Raynolds et al. 23] perform Nearest Neighbor Search (NNS) to handle loop closure and provide rotational correction. 
In hyperlapse video generation, Halperlin et al. [24] build a graph and a shortest path algorithm to help the selection of images before performing matches. While they do not call it a graph, Zelnik-Manor and Perona [25] use a weighted scheme in order to provide reliability information on features before matching them, where said weights are pure geometry-based metrics and not content-based, which reduces the chance of tackling difficult matching cases gracefully. Their method however presents strong capacities when it comes to its adopted formalized framework. Marzotto et al. 26] employ a global optimization scheme in order to optimize camera homographies using bundle adjustment algorithm, however their homographies are still based on features, hence limiting applicability to low textured document mosaicking.

Our main contributions concern:

- A confidence-driven, multiple image template matching solution for image mosaicking;

- A convenient formalized framework for dynamic global optimization;

- An experimental protocol for objective mosaicking quality assessment.

The paper is organized as follows. Section 2 gives the reader some basic tools and methodologies for images mosaicking. In Section 3 we detail all steps of our global framework. Section 4 presents experimental results and introduces our objective quality assessment protocol. Finally Section 5 concludes the paper.

\section{Materials and methods}

In order to make a self contained paper, we recall basic tools and the underlying mathematics used for global optimization in tile mosaicking.

Let's first recall the goal of our process: from a set of images, build a single large image using all the inputs in a way that no seam between images is visible. Figure 1 illustrates our goal from nine input images.

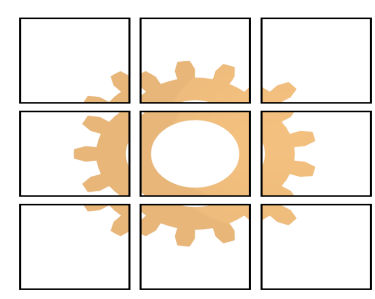

(a) Input images

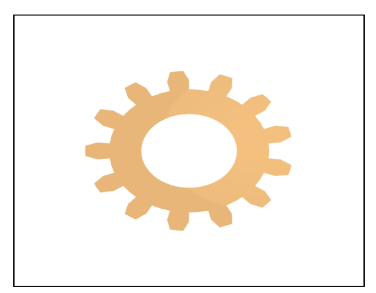

(b) Assembled image
Figure 1: Goal of the alignment process

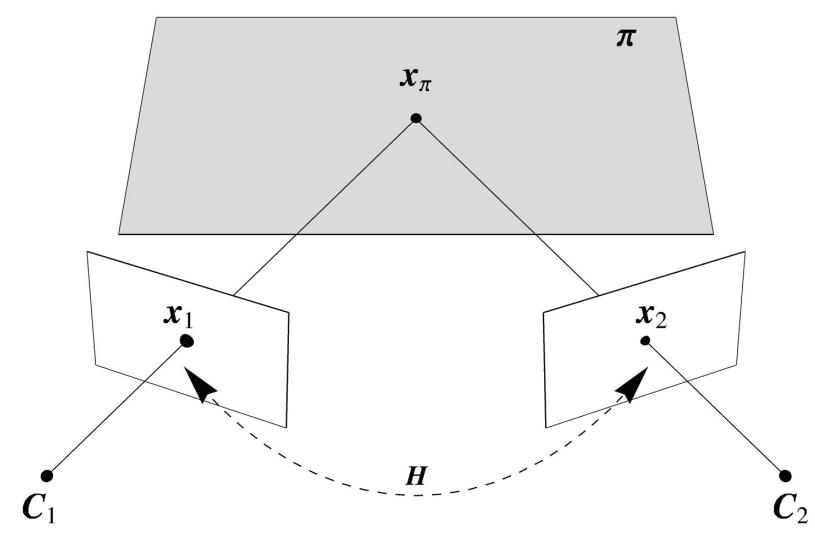

Figure 2: A planar homography $H$ is the transformation that maps points from an image to another image using a plane $\boldsymbol{\pi}$ as a reference.

\subsection{Acquisition process}

Generally speaking, tile scanning process can be summarized as the acquisition of an array $n \times m$ images. Each image has an associated camera and its associated pose. A pose is the relative orientation usually defined using a $3 \times 3$ rotation matrix and translation vector. Additionally, each camera is associated with an intrinsic matrix $K$ that, roughly speaking, encodes the lens parameters (focal, central point, ...) of the camera.

We can define the planar homography $H$ induced by a plane as the projective transformation that maps points from one camera to points of the other camera assuming that they are images of a point on a plane (See Figure 2). 
Let's define plane $\boldsymbol{\pi}$ by all the points $\boldsymbol{x}$ of $\mathbb{R}^{3}$ such that:

$$
\boldsymbol{n}^{\top} \boldsymbol{x}+d=0
$$

where $\boldsymbol{n}$ is the normal of the plane, and $d$ is distance of the plane to the origin of the reference frame.

Given two cameras $\mathcal{C}_{1}$ and $\mathcal{C}_{2}$, defined by their respective absolute rotations, centers and intrinsics $\left\{R_{1}, \boldsymbol{c}_{1}, K_{1}\right\}$ and $\left\{R_{2}, \boldsymbol{c}_{2}, K_{2}\right\}$, we can define the homography induced by the plane $\boldsymbol{\pi}$ as:

$$
H_{\boldsymbol{\pi}}=K_{2}\left(R_{2} R_{1}^{-1}-\frac{R_{2}\left(\boldsymbol{c}_{1}-\boldsymbol{c}_{2}\right) \boldsymbol{n}^{\top}}{d}\right) K_{1}^{-1} .
$$

In tile scanning, we assume that $K_{1}=K_{2}=I_{3}$, $R=R_{2} R_{1}^{-1}=I_{3}$ and plane is parallel to the cameras image planes (i.e. $\boldsymbol{n}=\boldsymbol{n}_{z}=(0,0,1)$ ). If we don't want to take into account translation in $z$ and assume that microscope image plane is parallel to the subject, we can further simplify and set $t_{z}=0$. Then the homography could be furthermore simplified (see Appendix A :

$$
H_{\boldsymbol{\pi}}=I_{3}-\frac{\boldsymbol{t} \boldsymbol{n}_{\boldsymbol{z}}^{\top}}{d}
$$

Where $I_{3}$ is the $3 \times 3$ identity matrix. Recall that $\boldsymbol{t} \boldsymbol{n}^{\top}$ is the outer product of $3 \mathrm{D}$ vectors:

$$
\boldsymbol{t n}^{\top}=\left[\begin{array}{c}
t_{x} \\
t_{y} \\
t_{z}
\end{array}\right]\left[n_{x}, n_{y}, n_{z}\right]=\left[\begin{array}{ccc}
t_{x} n_{x} & t_{x} n_{y} & t_{x} n_{z} \\
t_{y} n_{x} & t_{y} n_{y} & t_{y} n_{z} \\
t_{z} n_{x} & t_{z} n_{y} & t_{z} n_{z}
\end{array}\right]
$$

hence:

$$
\boldsymbol{t n}_{\boldsymbol{z}}^{\top}=\left[\begin{array}{ccc}
0 & 0 & t_{x} \\
0 & 0 & t_{y} \\
0 & 0 & 0
\end{array}\right]
$$

\subsection{Global homography optimization}

According to [27, we can define a global optimization process to estimate the set of homographies that best fit all camera views using neighboring links between subimages.

Given a camera $\mathcal{C}_{i}$, let $\mathcal{N}\left(\mathcal{C}_{i}\right)=\left\{C_{i}\right\}$ define all neighboring cameras capturing at least one part of its image content (i.e. having overlapping subimages). Let $H_{i j}$ be the homography between camera $\mathcal{C}_{i}$ and
$\mathcal{C}_{j}$. A global cost function between all homographies $\xi$ can be defined as:

$$
\xi\left(\left\{H_{i}\right\}\right)=\sum_{\mathcal{C}_{i}} \sum_{\mathcal{C}_{j} \in \mathcal{N}\left(\mathcal{C}_{i}\right)} w_{i j} \cdot \operatorname{dist}\left(H_{i}, H_{i j} H_{j}\right)^{2}
$$

where $w_{i j}$ is a weight that defines a level of trust or confidence given to the estimated link between camera $\mathcal{C}_{i}$ and camera $\mathcal{C}_{j}$, and $\operatorname{dist}(A, B)$ a distance measure between two homography matrices.

In global optimization mosaicking frameworks, a common goal is to estimate the best set of homographies $\left\{\hat{H}_{i}\right\}$ of $\left\{H_{i}\right\}$ from a given set of pairwise estimates $\left\{H_{i j}\right\}$ such that:

$$
\left\{\hat{H}_{i}\right\}=\underset{\left\{H_{i}\right\}}{\arg \min } \xi\left(\left\{H_{i}\right\}\right) .
$$

Many distance measures can be employed to evaluate cost function $\xi\left(\left\{H_{i}\right\}\right)$ in Equation 6. We rely on 28 and choose the square Frobenius norm defined as:

$\operatorname{dist}\left(H_{i}, H_{i j} H_{j}\right)^{2}=\operatorname{Tr}\left(\left(H_{i}-H_{i j} H_{j}\right)\left(H_{i}-H_{i j} H_{j}\right)^{\top}\right)$,

where difference $\left(H_{i}-H_{i j} H_{j}\right)$ can be evaluated using Equation 3

$$
\begin{aligned}
H_{i}-H_{i j} H_{j} & =\left(I_{3}-\frac{\boldsymbol{t}_{i} \boldsymbol{n}_{z}^{\top}}{d}\right)-\left(I_{3}-\frac{\boldsymbol{t}_{i j} \boldsymbol{n}_{z}^{\top}}{d}\right)\left(I_{3}-\frac{\boldsymbol{t}_{j} \boldsymbol{n}_{z}^{\top}}{d}\right) \\
& =-\frac{1}{d}\left(\boldsymbol{t}_{i}-\boldsymbol{t}_{j}-\boldsymbol{t}_{i j}\right) \boldsymbol{n}_{z}^{\top}-\frac{1}{d^{2}}\left(\boldsymbol{t}_{i j} \boldsymbol{n}_{z}^{\top}\right)\left(\boldsymbol{t}_{j} \boldsymbol{n}_{z}^{\top}\right) .
\end{aligned}
$$

As said above, we consider a scanning process which is parallel to the subject, which means the last coordinate of $\boldsymbol{t}_{i j}$ and $\boldsymbol{t}_{j}$ equals 0 , hence:

$$
-\frac{1}{d^{2}}\left(\boldsymbol{t}_{i j} \boldsymbol{n}_{z}^{\top}\right)\left(\boldsymbol{t}_{j} \boldsymbol{n}_{z}^{\top}\right)=0,
$$

and:

$$
H_{i}-H_{i j} H_{j}=-\frac{1}{d}\left(\boldsymbol{t}_{i}-\boldsymbol{t}_{j}-\boldsymbol{t}_{i j}\right) \boldsymbol{n}_{\boldsymbol{z}}^{\top},
$$

which finally gives us:

$$
\operatorname{dist}\left(H_{i}, H_{i j} H_{j}\right)^{2}=\frac{1}{d^{2}}\left\|\boldsymbol{t}_{i}-\boldsymbol{t}_{j}-\boldsymbol{t}_{i j}\right\|^{2} .
$$


Integrating this distance function into Equation 6 . and removing constant factor $\frac{1}{d^{2}}$ leads to the following definition for cost function $\xi($.$) :$

$$
\xi\left(\left\{H_{i}\right\}\right)=\sum_{\mathcal{C}_{i}} \sum_{\mathcal{C}_{j} \in \mathcal{N}\left(\mathcal{C}_{i}\right)} w_{i j} \cdot\left\|\boldsymbol{t}_{i}-\boldsymbol{t}_{j}-\boldsymbol{t}_{i j}\right\|^{2},
$$

\subsection{Pose-graph formalization}

Another way to formalize global image mosaicking optimization is to think of it in terms of a pose-graph optimization model, which is a framework of standard use in robot localization [29]. Here we sum up the building blocks of this methodology and show its strong similarity with the preceding formulation.

When scanning registration only relies on pairwise displacement estimates, mosaicking tends to suffer from several issues:

- The relative position between two cameras is imprecise because its accuracy is often limited;

- It is not reliable for poorly textured or textureless areas, for which position estimates suffer from an excessive number of possible solutions;

- In long paths, pairwise estimation errors can accumulate throughout the reconstruction process and result in large gaps between consecutive images at the end, a phenomenon known as drifting in video tracking literature.

One way to overcome such issues is to add constraints between subimages. In this context, the constrained global optimization problem is modeled after a weighted graph $G(V, E)$, where nodes (vertices) $\boldsymbol{p}_{i} \in V$ represent subimage positions and edges $z_{i j} \in E$ represent constraints between nodes $\boldsymbol{p}_{i}$ and $\boldsymbol{p}_{j}$. Each constraint is initially composed of measurements $\boldsymbol{p}_{i j}$ (pairwise estimates) and an information matrix $\Omega_{i j}$, encoding respectively the observed displacement between two nodes $\boldsymbol{p}_{i}$ and $\boldsymbol{p}_{j}$ and the level of reliability put into measurement $\boldsymbol{p}_{i j}$. Intuitively the later will indicate how strong an impact this constraint should have on the global optimization process. Figure 3 shows an example of a pose graph. Note that edges are oriented.

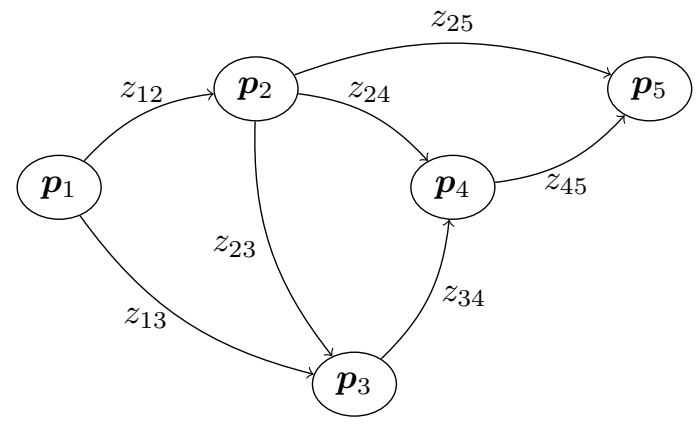

Figure 3: Example of a pose graph. $\boldsymbol{p}_{i}$ are the positions and $z_{i j}$ the constraints between positions.

Let $\boldsymbol{p}_{i j}$ be the actual distance between two nodes $\boldsymbol{p}_{i}$ and $\boldsymbol{p}_{j}$, that is the distance to be refined from an initial estimate $\tilde{\boldsymbol{p}}_{i j}$ :

$$
\boldsymbol{p}_{i j}=\boldsymbol{p}_{i}-\boldsymbol{p}_{j} .
$$

We define $\varepsilon\left(z_{i j}\right)=\varepsilon_{i j}$ as the estimation error of edge $z_{i j}$, computed from the difference between actual distance $\boldsymbol{p}_{i j}$ and measured distance $\tilde{\boldsymbol{p}}_{i j}$ as:

$$
\boldsymbol{\varepsilon}_{i j}=\left\|\Omega_{i j}\left(\boldsymbol{p}_{i j}-\tilde{\boldsymbol{p}}_{i j}\right)\right\|^{2},
$$

where information matrix $\Omega_{i j}$ serves as a convenience to introduce a reliability level into current estimate $\boldsymbol{p}_{i j}$, as explained earlier.

The global estimation error $\xi_{g}$ of graph $G(V, E)$ is defined as the sum of all errors $\varepsilon_{i j}$ computed on edges $z_{i j}$ :

$$
\begin{aligned}
\xi_{g}\left(\left\{\boldsymbol{p}_{i j}\right\}\right) & =\sum_{z_{i j} \in E} \boldsymbol{\varepsilon}_{i j} \\
& =\sum_{z_{i j}}\left\|\Omega_{i j}\left(\boldsymbol{p}_{i j}-\tilde{\boldsymbol{p}}_{i j}\right)\right\|^{2} \\
& =\sum_{z_{i j}}\left\|\Omega_{i j}\left(\boldsymbol{p}_{i}-\boldsymbol{p}_{j}-\tilde{\boldsymbol{p}}_{i j}\right)\right\|^{2} .
\end{aligned}
$$

This formation bears a strong resemblance with Equation 12. The main difference is that confidence weights are now defined as a matrix instead of a scalar. If we consider every measured distance to be as reliable along one displacement axis as it is along 
the other one (e.g. $x$ and $y$ axes on a $2 D$ image plane), we can write information matrix $\Omega_{i j}$ as :

$$
\Omega_{i j}=w_{i j} I_{2}
$$

with $I_{2}$ the identity matrix of size 2 . Combined with the above definition for $\Omega_{i j}$, Equation 15 now results exactly in the same optimization problem as the one defined by Equation 12. Therefore any optimization strategy, either pose-graph or classical optimization frameworks [30, 31] can be used to solve it. In our case we will use pose-graph optimization as a convenience for future works on non-rigid multi-image alignment. For this particular case we will have to refine subimage representation and geometric transform models, which pose-graph formalism handles gracefully.

\section{Calculation}

In this section, we provide details on our biomedical image mosaicking framework based on template matching and pose-graph optimization.

For the specific tile image scanning process we have to deal with, we assume that the fully reconstructed, large-field image compares itself with a matrix representation (aiming towards a graph) of $n \times m$ subimages. Let $\left(x_{i}, y_{i}\right)$ denote the pixel coordinates of the upper left corner of the $i$-th image within this matrix representation. Our goal is to estimate a best fitting position for all subimages such that their overlapping regions match as much as possible i.e. the seam between subimages is not visible. We also assume that subimages are acquired along a serpentine path, which in our case they are by design (and by mechanic rule). See Figure 4 for an overview. Such assumptions provide us with strong prior knowledge as to which subimage connects to one another.

In [27, Khurd et al. consider the 8 nearest neighbors for their optimization process. Here we only considered the 4 nearest neighbors, as in our case the overlapping area along a diagonal is never large enough to become a solid region for pairwise template matching.

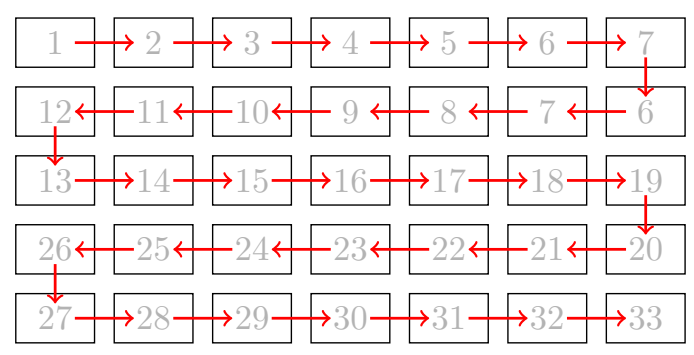

Figure 4: Tile scanning order. Images are acquired along a serpentine path.

\subsection{Pairwise matching and confidence}

The first building block of our framework consists in performing pairwise template matching. This step estimates initial displacement between two overlapping subimages. Template matching is a good trade-off between simpler 32] and computationally intensive methods 15] commonly used in medical imaging literature. Not only does template matching provide a fair initial estimation of subimage positions within the reconstructed image plane, but its computation steps reveal precious information about how well the alignment process went thus how reliable this estimate is. Given the appropriate use-case, in our case multi-photon micro-endoscopy, intensity-based template matching can be replaced by a similar but robust and cost-effective image alignment method described in [2].

Let $I_{i}$ and $I_{j}$ be two overlapping subimages extracted from the acquisition set, namely the source and target images. Template matching aims at estimating the position of $I_{j}$ with respect to $I_{i}$. Given a reasonable overlapping region between the two images, we define :

1. Search region $S_{i j}$ as the area of image $I_{i}$ where Template $T_{i j}$ should be searched for ;

2. Template $T_{i j}$ as the part of image $I_{j}$ to look for in Search region $S_{i j}$.

Search region $S_{i j}$ is located within the overlapping area of $I_{i}$ and $I_{j}$. Its size is to be defined as slightly larger than the size of the estimated overlap in order to produce an error margin for imprecise scans. In 

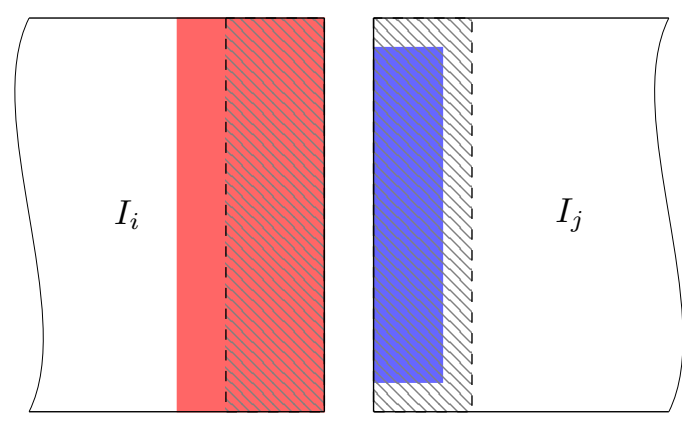

Figure 5: Pairwise template matching : theoretical overlapping area in dashed gray, search region $S_{i j}$ of $I_{i}$ in red, template $T_{i j}$ of $I_{j}$ in blue.

a similar fashion, template $T_{i j}$ is defined as a part of $I_{j}$ located within the same overlapping area. It is smaller than said area in order to produce an error margin as well. This is illustrated on Figure 5 .

Assuming a subimage $I_{i}$ of size $W \times H$ and a template $T_{i j}$ of size $w \times h$, template matching computes a scoring function $\mathcal{C}_{i j}(x, y)$ defined within a $(W-w+1) \times(H-h+1)$ support defined as the query region in the following. Function $\mathcal{C}_{i j}(x, y)$ should evaluate and score all possible displacements $(x, y)$ that are likely to align image $I_{i}$ with image $I_{j}$. We decided to use the zero mean normalized cross correlation defined as:

$$
\mathcal{C}_{i j}(x, y)=\frac{\sum_{u} \sum_{v}\left(T_{i j}^{\prime}(u, v) \cdot S_{i j}^{\prime}(u+x, v+y)\right)}{\sqrt{\sum_{u} \sum_{v} T_{i j}^{\prime}(u, v)^{2} \cdot S_{i j}^{\prime}(u, v)^{2}}}
$$

with:

$$
\begin{aligned}
& S_{i j}^{\prime}(x, y)=S_{i j}(x, y)-\frac{1}{W \cdot H} \sum_{u} \sum_{v} S_{i j}(u, v) \\
& T_{i j}^{\prime}(x, y)=T_{i j}(x, y)-\frac{1}{w \cdot h} \sum_{u} \sum_{v} T_{i j}(u, v) .
\end{aligned}
$$

Should function $\mathcal{C}_{i j}(x, y)$ admit only one maximum, the corresponding point over its definition domain is to be considered as the theoretical best matching position. Whether such a point exists or not, the distribution and/or geometry of $\mathcal{C}_{i j}(x, y)$ provides precious information about the level of trust we can put into said position estimate. For the sake

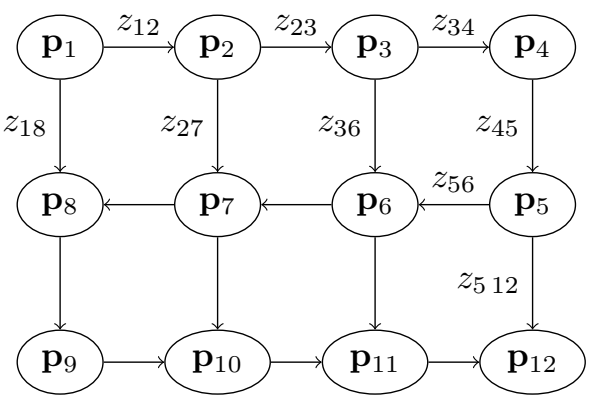

Figure 6: Pose-graph topology

of simplification we consider template $T_{i j}$ to be anchored at the origin of image $I_{j}$ i.e. top left corner $(0,0)$. There exists a bijection between any test position $(x, y)$ and the relative motion $\left(\Delta_{x}, \Delta_{y}\right)$ of template $T_{i j}$ with respect to source image $I_{i}$ :

$$
\begin{aligned}
\left(\Delta_{x}, \Delta_{y}\right) & =\mathcal{M}_{i j}(x, y) \\
(x, y) & =\mathcal{M}_{i j}^{-1}\left(\Delta_{x}, \Delta_{y}\right) .
\end{aligned}
$$

The cost value for any position $(x, y)$ can be evaluated, and the highest one $\mathcal{C}_{i j}\left(\tilde{x}_{i j}, \tilde{y}_{i j}\right)$ indicates either best matching position $\left(\tilde{x}_{i j}, \tilde{y}_{i j}\right)$ or best displacement $\left(\tilde{\Delta}_{i j_{x}}, \tilde{\Delta}_{i j_{y}}\right)$ :

$$
\begin{aligned}
\left(\tilde{x}_{i j}, \tilde{y}_{i j}\right) & =\underset{(x, y)}{\arg \max } \mathcal{C}_{i j}(x, y) \\
\left(\tilde{\Delta}_{i j_{x}}, \tilde{\Delta}_{i j_{y}}\right) & =\mathcal{M}_{i j}\left(\tilde{x}_{i j}, \tilde{y}_{i j}\right) .
\end{aligned}
$$

Note that at this stage all optimal estimates are denoted with tildes. These will be refined later on to be finally denoted with hats, as mathematical conventions suggest and as seen in Equation 7.

\subsection{Global dynamic optimization}

We refer to pairwise matches in order to build an initial pose-graph from the set of images. The induced graph topology for initial pairwise template matching along a serpentine path is illustrated on Figure 6. Extra pairwise matchings (i.e. extra constraints) are performed using 4-neighbors connectivity information to refine the graph with new connections. 
Graph edges now define a set of constraints $\left\{z_{i j}\right\}$ between all subimages.

As stated in Section 2, pairwise template matches performed along the acquisition path alone are not reliable enough to ensure good large-field image reconstructions over long image sequences or when dealing with low-textured areas. Moreover, extra pairwise matches induced by nearest neighbourhood search strategies leave us with a potentially over-constrained optimization problem to solve. The pose graph topology then becomes a convenient framework to solve this problem.

Refining pose graph nodes, i.e. solving the overconstrained optimization problem for optimal image reconstruction, consists in finding the best compromising set of positions $\left\{\boldsymbol{p}_{i}\right\}$, preferably using weighting scalars $\left\{w_{i j}\right\}$ (Equation 18 and/or Equation 6) as a mean to inject extra information such as confidence levels into this process. Having a set of constant values $w_{i j}=1$ could be a starting point [27] and the resulting solution is likely to bear resemblance with one computed through classical Minimum Mean Square Error (MMSE) estimation. However erroneous pairwise matches are likely to appear in our set of initial estimates $\tilde{\boldsymbol{p}}_{i j}$ (see Section 2.3) and we want to penalize them.

For obvious reasons, we could define the reliability of an initial estimate $\tilde{\boldsymbol{p}}_{i j}$ as having a direct relationship with the maximum of normalized matching cost function $\mathcal{C}_{i j}(x, y)$, hence:

$$
w_{i j}=w_{c_{i j}}=\mathcal{C}_{i j}\left(\tilde{x}_{i j}, \tilde{y}_{i j}\right) \text {, }
$$

where $w_{c_{i j}} \in[0,1]$.

We name this first approach Constant weighting, as opposed to the previous Unit weighting mentioned above. In our experiments it yields better results than Unit weighting. However it should be noted that it only relies on a unique evaluation of cost matching function $\mathcal{C}_{i j}(x, y)$. As explained earlier, function $\mathcal{C}_{i j}(x, y)$ may admit not just a single maximum but several, making estimate $\left(\tilde{x}_{i j}, \tilde{y}_{i j}\right)$ just a random selection among a set of probable candidates. Furthermore, depending on how this maximum of $\mathcal{C}_{i j}(x, y)$ is estimated, $\left(\tilde{x}_{i j}, \tilde{y}_{i j}\right)$ may also correspond to a local maximum instead of a global one. This is a common cause of failure with wide-spread convex optimization algorithms.

We mentioned that the distribution and/or geometry of $\mathcal{C}_{i j}(x, y)$ provides precious information about the level of trust we can put into a position estimate. Now we introduce a second term to Equation 26 in order to refine the analysis of function $\mathcal{C}_{i j}($.$) . Instead$ of evaluating global statistics, we will simply evaluate the new values of $\mathcal{C}_{i j}($.$) for updated estimations$ $\left(x_{i j}, y_{i j}\right)$, where $\left(\tilde{x}_{i j}, \tilde{y}_{i j}\right)$ is passed as the initial solution to the solving problem that lies upon the posegraph topology. The idea lying underneath this new term of Dynamic weighting is that some matches will tolerate imprecision better than others. Using this as a computation rule, we take advantage of the posegraph iterative optimization process to introduce a dynamic adjustment of confidence weights:

$$
w_{d_{i j}}=-\mathcal{C}_{i j}\left(x_{i j}, y_{i j}\right),
$$

where $w_{d_{i j}} \in\left[-\mathcal{C}_{i j}\left(\tilde{x}_{i j}, \tilde{y}_{i j}\right), 0\right]$ and $\left(x_{i j}, y_{i j}\right)$ denote the updated weight and displacement from initial point estimate $\left(\tilde{x}_{i j}, \tilde{y}_{i j}\right)$, said parameters being computed from the current iteration in the pose-graph estimation of $\boldsymbol{p}_{i j}=\boldsymbol{p}_{i}-\boldsymbol{p}_{j}$.

Dynamic weight $w_{d_{i j}}$ adjusts a constant weight $w_{c_{i j}}$ given the cost value of a current pose-graph position estimate. It will penalize high values of $\mathcal{C}_{i j}\left(x_{i j}, y_{i j}\right)$, as the closer they are to $\mathcal{C}_{i j}\left(\tilde{x}_{i j}, \tilde{y}_{i j}\right)$, the more difficult it becomes to decide whether the optimal position is actually $\left(\tilde{x}_{i j}, \tilde{y}_{i j}\right)$ or $\left(x_{i j}, y_{i j}\right)$. In this case we want to consider connection $z_{i j}$ as unreliable and reduce weight $w_{i j}$. On the other side, low values of $\mathcal{C}_{i j}\left(x_{i j}, y_{i j}\right)$ would indicate a strong confidence to put into $\left(\tilde{x}_{i j}, \tilde{y}_{i j}\right)$ or at least in $\mathcal{C}_{i j}\left(\tilde{x}_{i j}, \tilde{y}_{i j}\right)$. In that case we want to let constant weight $w_{c_{i j}}$ to operate alone with the pose-graph optimization process.

Pose-graph edge weights $w_{i j}$ are finally computed $\mathrm{a}$

\footnotetext{
${ }^{1}$ Implementation notes: should current estimate $\left(x_{i j}, y_{i j}\right)$ locate out the definition domain of $\mathcal{C}_{i j}(),. w_{d_{i j}}$ will be set to $-\mathcal{C}_{i j}\left(\tilde{x}_{i j}, \tilde{y}_{i j}\right)$, as it indicates a position located outside the overlapping area between $I_{i}$ and $I_{j}$. If using Fourier transformbased solutions for template matching, an efficient computation of $w_{d_{i j}}$ will require saving pre-computed discrete cost functions $\mathcal{C}_{i j}(x, y)$ i.e. correlation maps.
} 


$$
w_{i j}=w_{c_{i j}}+\alpha \cdot w_{d_{i j}},
$$

where a constant value $\alpha \in[0,1]$ is introduced in order to control the influence of dynamic weighting and make Equation 26 a particular case of Equation 28 .

Once the last refinement iteration is over, current thus optimal estimates $\left(x_{i j}, y_{i j}\right)$ can be denoted $\left(\hat{x}_{i j}, \hat{y}_{i j}\right)$ and all optimal pose-graph nodes $\left\{\hat{\boldsymbol{p}}_{i}\right\}$ provide us with the best subimage positions for reconstruction.

\section{Results and Discussion}

In this section, we present experimental results obtained with our mosaicking framework and compare all three weighting methods presented in Section 3 namely Unit weighting, Constant weighting and Dynamic weighting. We first present results obtained from actual tile scanned biomedical data for visual (subjective) assessment, then we introduce a new strategy for objective matching quality assessment. Using either of the two evaluation methods, our posegraph-based Dynamic weighting contribution yields better results than the other two.

The subimages we used were extracted from microscopic tile scans of biological tissues. They come from a set of scans acquired for biopsy of transplanted human kidneys and tumor histology. We were given prior knowledge from mechanical and optical calibration about the scanning path and the approximate size of and overlap between subimages.

Implementation was made in $\mathrm{C}++$ using OpenCV 33 for template matching computation and Ceres 30 as the global optimization solver.

\subsection{Subjective visual quality assess- ment}

First we provide results obtained from simple pairwise template matching and compare these results with the ones obtained using global constraints and optimization. Then we focus on the advantages of Dynamic weighting.
Four mosaicking methods were tested :

- Sole pairwise matching: alignment estimation using only template matching on image pairs along the acquisition path;

- Global matching with Unit weighting : relies on global optimization using 4-connected images pairs and all weighting scalars set to 1 ;

- Global matching with Constant weighting : weighting scalars are set to the highest pairwise matching scores (Equation 26 or Equation 28 with $\alpha=0$ );

- Global matching with Dynamic weighting : weighting scalars are set to the highest pairwise matching scores and regulated for each iteration with matching scores of current estimates (Equation 28 with $\alpha=1$ ).

Sole pairwise matching The first set of reconstructed images demonstrate why global matching is a fundamental step in our application. Figure 7 shows two examples of Sole pairwise matching mosaicking. While Figure 7 presents a tolerable reconstruction, Figure $7 \mathrm{~b}$ does not. In both examples, failure is mainly the consequence of large blank or low-textured regions over the large-field target plane.

Global matching with unit weighting Global optimization as a whole largely improves reconstruction results when individual pairwise matches fail. As an example, Figure 7 a was improved using Global matching with Unit weighting to a more satisfying result show on Figure 8 .

Global matching with constant weighting Global matching with Constant weighting forces important nodes to be considered accurately. Figure 9 shows significant reconstruction quality improvement from Unit weighting (left) to Constant weighting (right), especially when looking at straight edges and/or curved contours. While we can decipher tiles on Unit weighting reconstruction, Constant weighting produces a smoother overlap. We should note however that some artefacts are still visible (e.g. 


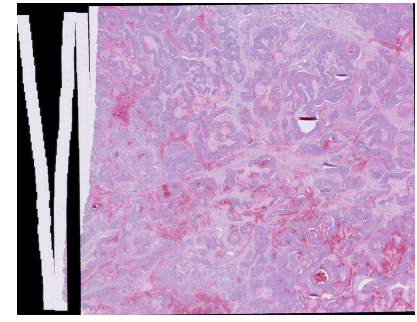

(a)

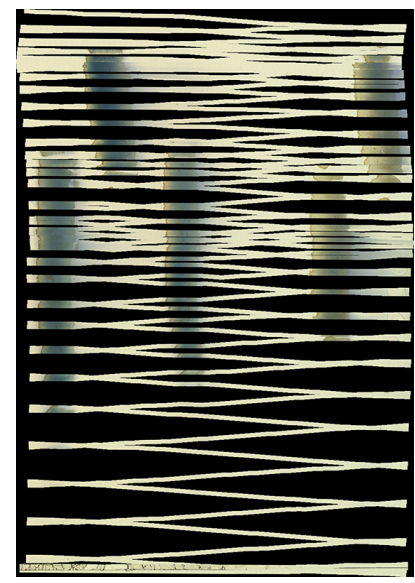

(b)

Figure 7: Examples of failed reconstruction using only pairwise template matching

Figure 9ff) and will rely on Dynamic weighting for better accuracy.

Global matching with dynamic weighting Now we show proofs of improvement using Dynamic weighting. Figure 11 compares Constant weighting (a and c) with Dynamic weighting (b and d). As we can see, strong tiling artifacts that remain even when using Constant weighting tend to disappear with $D y$ namic weighting estimations.

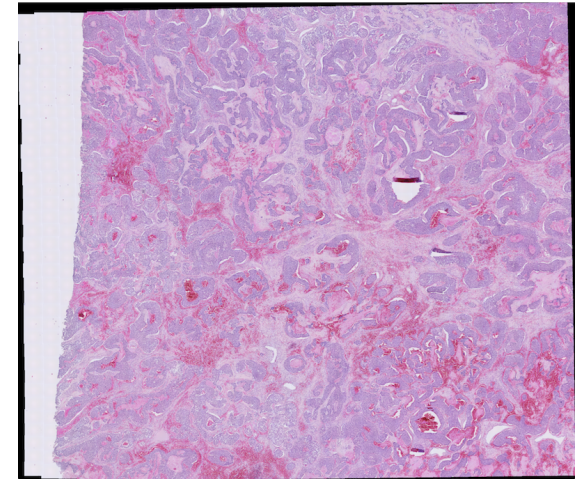

Figure 8: Global matching with Unit weighting on the same set of subimages as the one used for Figure 7 (a)

\subsection{Comparison of matchings}

\subsection{Comparison of a full with refer- ence algorithms}

To compare our approach with the litterature, some stitching algorithms are available. For example, we can find MicroMos[20], MIST[21], the grid stitching method by [17] or Terastitcher [19].

These methods have limits on the memory use or on the number of titles which composes a mosaic. MIST has been developped with a performance objective for well-known sofware Image $2^{2}$ in Java programming language and mosaic from high resolution subimages can so be reconstructed.

Original images are of size 2456 by 1842 pixels and the scanner system acquires tiles with a maximal horizontal and vertical overlap equal to $8 \%$. We illustrate performances of our method with three sequences:

- the first sequence is composed of $30 \times 12$ subimages. In Figure 12 (a), the mosaic reconstructed by MIST has a resolution equal to $53211 \times 29323$ and the one of our method in Figure $12(\mathrm{~b})$ $52120 \times 29061$ pixels.

- the second sequence is composed of $17 \times 30$ subimages. In Figure 13(a), the mosaic reconstructed by MIST has a resolution equal to

\footnotetext{
${ }^{2}$ https://imagej.nih.gov/ij/
} 


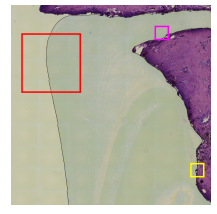

(a)

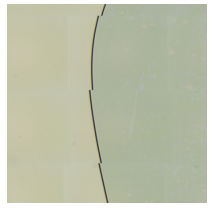

(c)

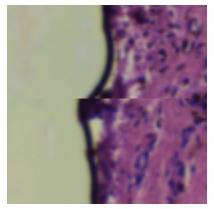

(e)

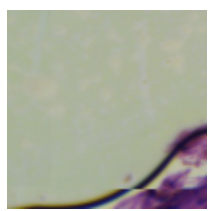

(g)

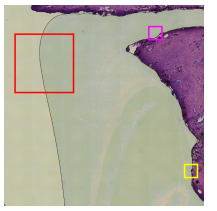

(b)

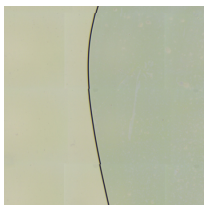

(d)

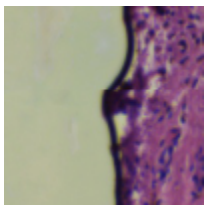

(f)

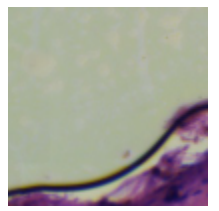

(h)
Figure 9: A visual comparison between Unit weighting (left) and Constant weighting (right) shows that significantly better results are obtained when using Constant weighting

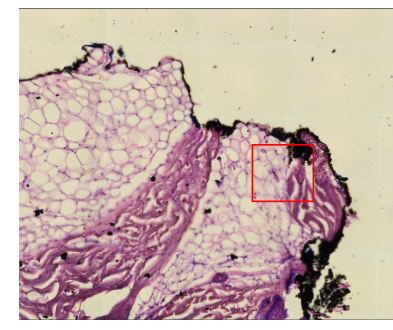

(a)

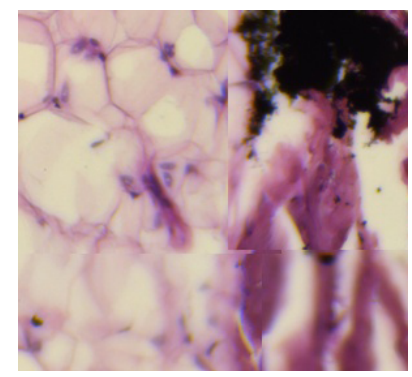

(c)

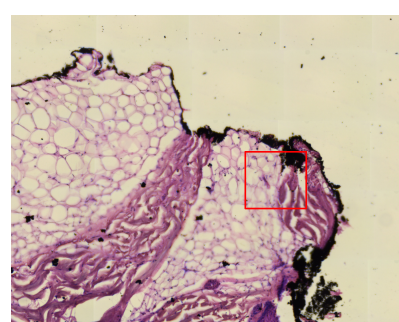

(b)

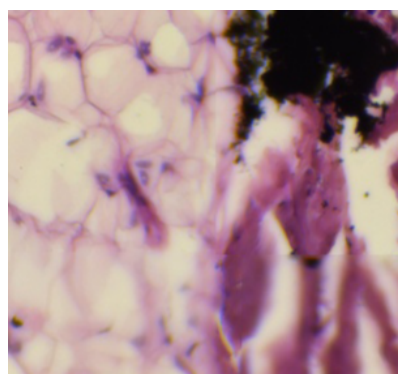

(d)
Figure 10: Comparison between Constant weighting and Dynamic weighting: (a) (c) Constant weighting; (b) (d) Dynamic weighting. (c) and (d) zoomed-in over the red rectangle. 


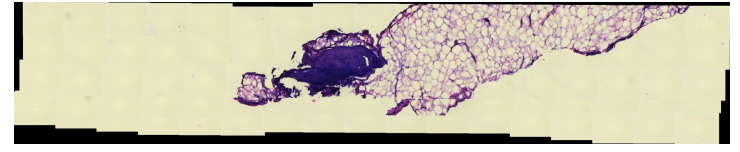

(a)

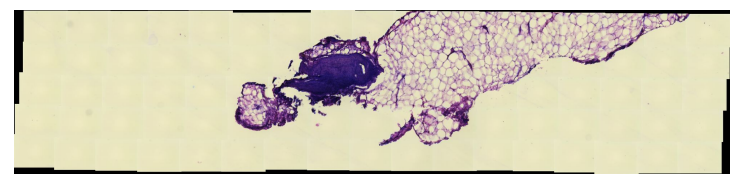

(b)

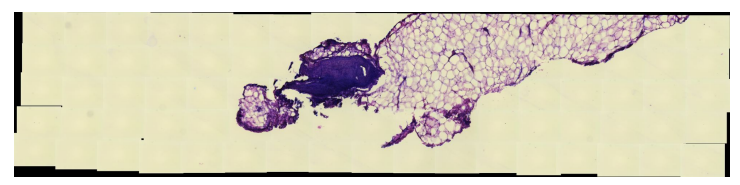

(c)

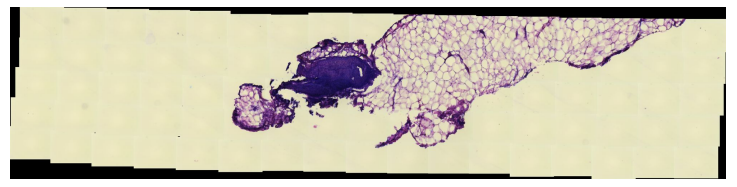

(d)

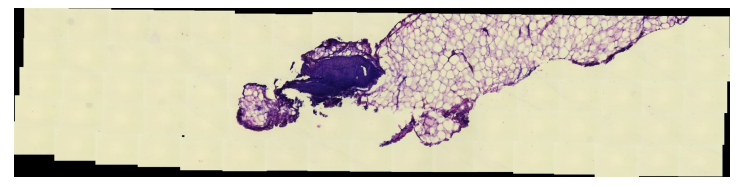

(e)

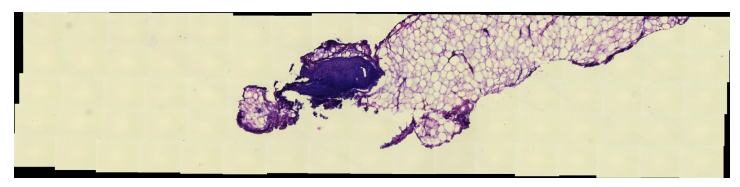

(f)

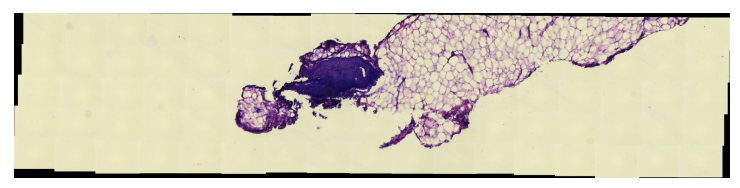

(g)

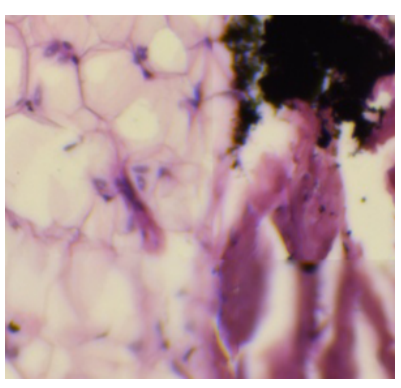

(h)

Figure 11: Comparison between Constant weighting and Dynamic weighting: (a) (c) Constant weighting; (b) (d) Dynamic weighting. (c) and (d) zoomed-in

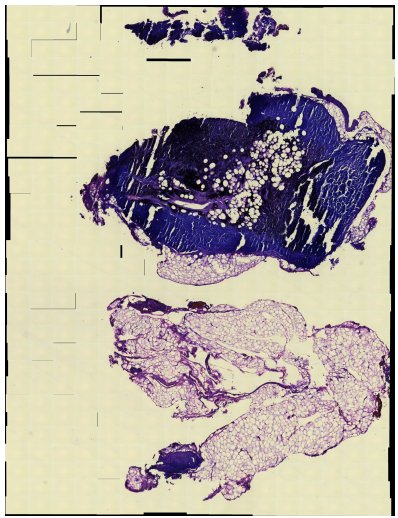

(a)

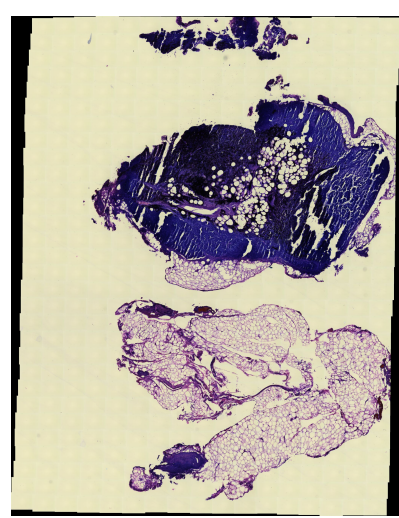

(b)
Figure 12: Comparison between mosaic with (a) MIST and (b) proposed dynamic weighting method.

\begin{tabular}{c||c|c|c} 
& MIST & Constant & Dynamic \\
\hline \hline Relative displacement & 93,40 & 68.35 & 69.04 \\
Global optimization & 130.93 & 0.010 & 23.08 \\
Stitching, image creation & 51.69 & 24.72 & 26.6 \\
\hline Total & 276.02 & 93.08 & 118.72
\end{tabular}

Table 1: Computation time (in seconds) on the first sequence composed of $30 \times 12$ subimages

$52884 \times 40332$ and the one of our method in Figure 13 (b) $52768 \times 41188$ pixels.

- the third sequence is composed of $15 \times 25$ subimages. In Figure 14(a), the mosaic reconstructed by MIST has a resolution equal to $59286 \times 26859$ and the one of our method in Figure 14(b) $59772 \times 26620$ pixels.

These results highlight that our method outperforms MIST, the reference of mosaicking algorithms. We ensure a perfect registration without sewing problem (black lines on Figures 12(a), 13(a) and 14(a) with MIST)

The limitations encountered in the stitching method evaluation leads us to propose an objective quality assessment of the mosaicking framework.

In Table 1. we illustrate the computation time of the reconstruction of the first sequence which is composed of $30 \times 12=360$ images of resolution 


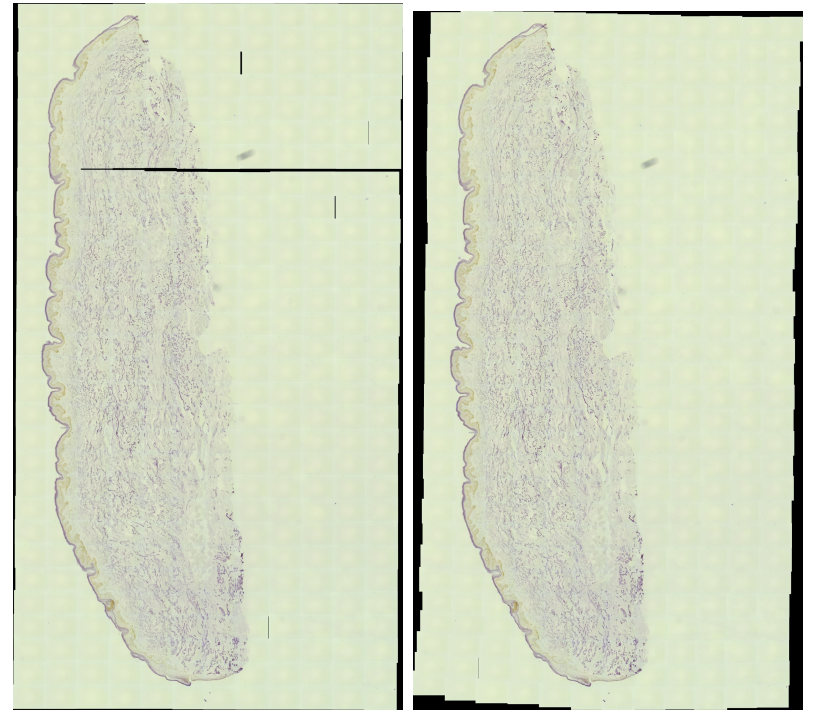

(a)

(b)

Figure 13: Comparison between mosaic with (a) MIST and (b) proposed dynamic weighting method.

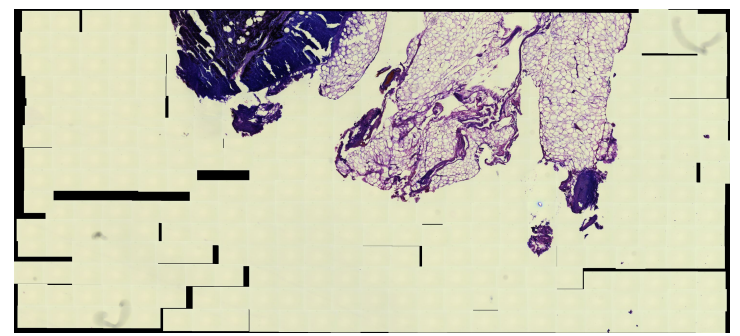

(a)

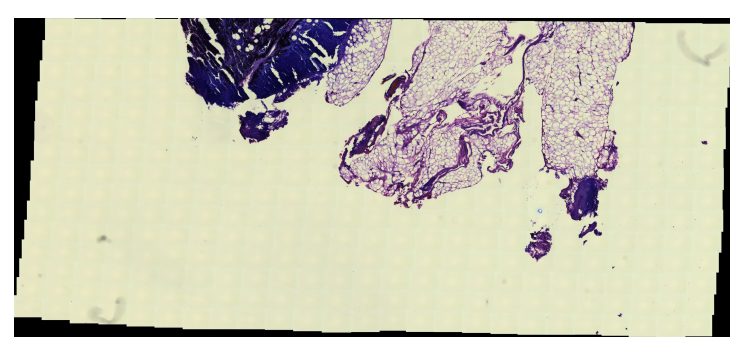

(b)

Figure 14: Comparison between mosaic with (a) MIST and (b) proposed dynamic weighting method.
$2456 \times 1842$. On Intel Xeon CPU E5-2650 at $2.3 \mathrm{GHz}$, $64 \mathrm{~GB}$ of memory and a Linux system, the computation of our method is more than 2.3 times faster than MIST. Note that our method have not been parallelized or implemented with CUDA.

\subsection{Objective quality assessment}

While the above experiments show significant improvement on a subjective level, the original sets of image data are solely made of raw pixel values and not actual position metadata. As a result, we cannot rely on them to compute distance metrics or anything else that would provide us with an objective quality assessment of our mosaicking framework. We were however able to manage such an evaluation using said image data and modeling software Blender [34 to generate a ground truth data with position information.

Ground truth data consists in a virtual plane with a texture associated to it. We chose high resolution textures using OpenSlide samples 35, as they share common content with the actual images that were acquired for original our dataset. Within Blender we define an acquisition path for a virtual camera in order to simulate a scanning process (Figure 15). All path parameters are controlled: number of subimages in a row, number of subimages in a column and overlaps. Camera positions and projected subimage positions are recorded as well, serving as reference material for position error computations.

Now we can perform mosaicking and try to recover the (supposedly unknown) virtual camera path from raw image data. Distance (mean square) errors in pixel coordinates between ground truth camera positions and the estimated ones were computed from three different synthetic scenes $C M U-1, O S-1$ and Leica-1. Results are shown on Table 2.

Results for all three scenes are constant, with Unit weighting always having the worst results, then Constant weighting showing significant improval, and Dynamic weighting yielding the best performance. Such observations were already perceptible on the subjective quality assessments above. They clearly show the rationale for confidence-driven estimations and the relevance of dynamic weighting. 


\section{Conclusion}

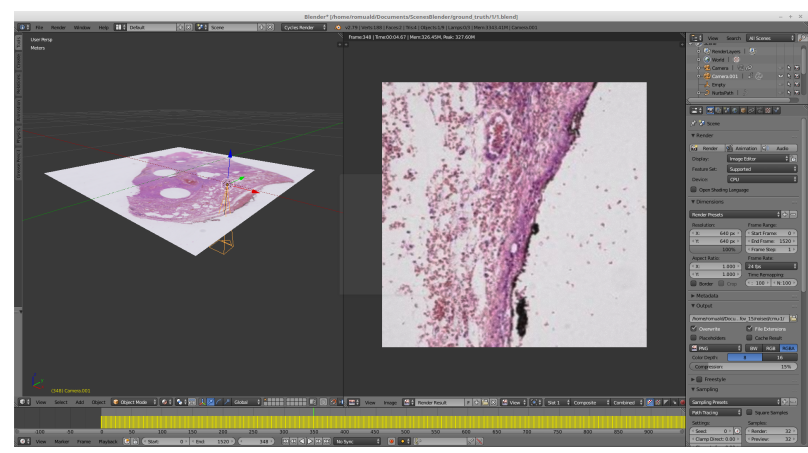

Figure 15: Ground truth data generation for objective quality assessment using Blender

\begin{tabular}{|l||c|c|}
\hline \multicolumn{3}{|c|}{ Unit weighting } \\
\hline Scene & Mean MSE & Max MSE \\
\hline CMU-1 & 14.7 & 53.6 \\
OS-1 & 6.27 & 57 \\
Leica-1 & 22.0 & 73 \\
\hline \multicolumn{3}{|c|}{ Constant weighting } \\
\hline Scene & Mean MSE & Max MSE \\
\hline CMU-1 & 9.5 & 49.5 \\
OS-1 & 2.25 & 53 \\
Leica-1 & 13.28 & 40 \\
\hline \multicolumn{3}{|c|}{ Dynamic weighting } \\
\hline Scene & Mean MSE & Max MSE \\
\hline CMU-1 & $\mathbf{9 . 3}$ & 44.7 \\
OS-1 & $\mathbf{2 . 1 8}$ & 53 \\
Leica-1 & $\mathbf{1 2 . 5 5}$ & 40 \\
\hline \hline
\end{tabular}

Table 2: Relative mean square displacement errors (MSE) in pixel units using Unit weighting, Constant weighting and Dynamic weighting. For every image scene, Dynamic weighting show the best performance.
In this paper, we have presented a global, confidencedriven optimization framework to perform mosaicking on biomedical images. Starting from an initial set of pairwise subimage matches, our method reduces positioning errors caused by incorrect matches due to poor texture representations, hence increasing visual quality of the overall image. Our main contribution to this framework is the introduction of confidencerelated constraints as weighting values between two nodes of a pose-graph. As every edge can iteratively evaluated for reliability, the pose-graph becomes a convenient formalized framework for dynamic optimization. In our case confidence values are computed from pairwise matching scores.

Results on real world biomedical data show that our method performs well and helps to reduce small misalignments between images. However, these are subjective assumptions based on aesthetic criteria. Another contribution in this paper was to enforce such observations with a new validation strategy for objective image reconstruction quality assessment. Still using real biomedical data as images sources, we have generated annotated ground truth subimages (i.e. subimages with position information) within the Blender 3D rendering software to simulate camera motion over an image plane. Distance errors between ground truth positions and the estimated ones were computed and show significant improvement as well when using our dynamic optimization method.

An important aspect of using the pose-graph framework is that it can handle many constraints in a straightforward manner. In future works, we will address the issue of complex rigid and nonrigid motion and deformations between pairwise subimages, using either parametric (e.g. BSplines) or non-parametric (e.g. Daemons) models for deformation. We expect the pose-graph framework to deal with such a multimodel data approximation gracefully.

\section{A Relative motion}

The homography induced by the plane $\boldsymbol{\pi}$ (Equation 2 could be simplified if we assume first camera 
is at origin and with identity rotation. In that case, we can define the rotation $R$ and the translation $t$ of $\mathcal{C}_{2}$ with respect to $\mathcal{C}_{1}$. is:

The relative motion $\{R, t\}$ of $\mathcal{C}_{2}$ with respect to $\mathcal{C}_{1}$

$$
\begin{aligned}
R & =R_{2} R_{1}^{-1}, \\
\boldsymbol{t} & =\boldsymbol{t}_{2}-R \boldsymbol{t}_{1} .
\end{aligned}
$$

Given that:

$$
\begin{aligned}
& \boldsymbol{t}_{1}=-R_{1} \boldsymbol{c}_{1}, \\
& \boldsymbol{t}_{2}=-R_{2} \boldsymbol{c}_{2},
\end{aligned}
$$

with $\boldsymbol{c}_{1}$ and $\boldsymbol{c}_{2}$ respectively, the center of the cameras $\mathcal{C}_{1}$ and $\mathcal{C}_{2}$. Then we have:

$$
\begin{aligned}
\boldsymbol{t} & =\boldsymbol{t}_{2}-R \boldsymbol{t}_{1} \\
& =-R_{2} \boldsymbol{c}_{2}-R_{2} R_{1}^{-1}\left(-R_{1} \boldsymbol{c}_{1}\right) \\
& =-R_{2} \boldsymbol{c}_{2}+R_{2} \boldsymbol{c}_{1} \\
& =R_{2}\left(\boldsymbol{c}_{1}-\boldsymbol{c}_{2}\right) .
\end{aligned}
$$

Hence, Equation 2 could be rewritten as:

$$
H_{\boldsymbol{\pi}}=K_{2}\left(R-\frac{\boldsymbol{t n}^{\top}}{d}\right) K_{1}^{-1} .
$$

\section{Conflicts of interest}

The authors state no conflict of interest and have nothing to disclose.

\section{References}

[1] G. Ducourthial, P. Leclerc, T. Mansuryan, M. Fabert, J. Brevier, R. Habert, F. Braud, R. Batrin, C. Vever-Bizet, G. Bourg-Heckly, et al., Development of a real-time flexible multiphoton microendoscope for label-free imaging in a live animal, Scientific reports 5 .

[2] P. Bourdon, D. Helbert, A data-driven approach to feature space selection for robust microendoscopic image reconstruction, in: IEEE International Conference on Image Processing (ICIP), 2017.
[3] N. Pham, D. Helbert, P. Bourdon, P. Carré, Spectral graph wavelet based nonrigid image registration, in: 2018 25th IEEE International Conference on Image Processing (ICIP), IEEE, 2018, pp. 3348-3352.

[4] N. Pham, D. Helbert, P. Bourdon, P. Carré, Image registration for biomedical images on irregular grids, in: CoRESA, 2018.

[5] F. P. Oliveira, J. M. R. Tavares, Medical image registration: a review, Computer methods in biomechanics and biomedical engineering 17 (2) (2014) 73-93.

[6] A. P. James, B. V. Dasarathy, Medical image fusion: A survey of the state of the art, Information Fusion 19 (2014) 4-19.

[7] T. Bergen, T. Wittenberg, Stitching and surface reconstruction from endoscopic image sequences: a review of applications and methods, IEEE journal of biomedical and health informatics 20 (1) (2016) 304-321.

[8] D. Makovoz, F. R. Marleau, Point source extraction with MOPEX, Publ. Astron. Soc. Pac. 117 (2005) 1113-1128. arXiv:astro-ph/0507007, doi:10.1086/432977

[9] S. Nagaraja, C. J. Prabhakar, P. U. P. Kumar, Parallax effect free mosaicing of underwater video sequence based on texture features, CoRR abs/1411.2090.

[10] D. G. Lowe, Object recognition from local scaleinvariant features, in: Computer vision, 1999. The proceedings of the seventh IEEE international conference on, Vol. 2, Ieee, 1999, pp. 11501157.

[11] T. Köhler, A. Heinrich, A. Maier, J. Hornegger, R. P. Tornow, Super-resolved retinal image mosaicing, in: 2016 IEEE 13th International Symposium on Biomedical Imaging (ISBI), 2016, pp. 1063-1067. doi:10.1109/ISBI. 2016.7493449

[12] L. Zhang, M. Ye, P. Giataganas, M. Hughes, G.-Z. Yang, Autonomous scanning for endomicroscopic mosaicing and 3d fusion, 2017 IEEE 
International Conference on Robotics and Automation (ICRA) (2017) 3587-3593.

[13] R. E. Kalman, et al., A new approach to linear filtering and prediction problems, Journal of basic Engineering 82 (1) (1960) 35-45.

[14] R. Miranda-Luna, C. Daul, W. C. Blondel, Y. Hernandez-Mier, D. Wolf, F. Guillemin, Mosaicing of bladder endoscopic image sequences: Distortion calibration and registration algorithm, IEEE Transactions on Biomedical Engineering 55 (2) (2008) 541-553.

[15] P. Viola, W. M. Wells III, Alignment by maximization of mutual information, International journal of computer vision 24 (2) (1997) 137154.

[16] T. Vercauteren, A. Perchant, G. Malandain, X. Pennec, N. Ayache, Robust mosaicing with correction of motion distortions and tissue deformations for in vivo fibered microscopy, Medical image analysis 10 (5) (2006) 673-692.

[17] S. Preibisch, S. Saalfeld, P. Tomancak, Globally optimal stitching of tiled 3d microscopic image acquisitions, Bioinformatics 25 (11) (2009) 1463-1465.

[18] K. E. Loewke, D. B. Camarillo, W. Piyawattanametha, M. J. Mandella, C. H. Contag, S. Thrun, J. K. Salisbury, In vivo micro-image mosaicing, IEEE Transactions on Biomedical Engineering 58 (1) (2011) 159-171.

[19] A. Bria, G. Iannello, Terastitcher - a tool for fast automatic 3d-stitching of teravoxel-sized microscopy images, BMC Bioinformatics 13 (1) (2012) 316. doi:10.1186/1471-2105-13-316. URL https://doi.org/10.1186/ 1471-2105-13-316

[20] F. Piccinini, A. Bevilacqua, E. Lucarelli, Automated image mosaics by non-automated light microscopes: the micromos software tool, Journal of microscopy 252 (3) (2013) 226-250.
[21] J. Chalfoun, M. Majurski, T. Blattner, K. Bhadriraju, W. Keyrouz, P. Bajcsy, M. Brady, Mist: Accurate and scalable microscopy image stitching tool with stage modeling and error minimization, Scientific reports 7 (1) (2017) 4988.

[22] K. Kose, M. Gou, O. Yélamos, M. Cordova, A. M. Rossi, K. S. Nehal, E. S. Flores, O. Camps, J. G. Dy, D. H. Brooks, et al., Automated videomosaicking approach for confocal microscopic imaging in vivo: an approach to address challenges in imaging living tissue and extend field of view, Scientific reports 7 (1) (2017) 10759.

[23] M. Reynolds, T. S. F. Haines, G. J. Brostow, Swipe Mosaics from Video, arXiv:1609.08080 [cs]ArXiv: 1609.08080.

URL http: //arxiv .org/abs/1609.08080

[24] T. Halperin, Y. Poleg, C. Arora, S. Peleg, EgoSampling: Wide view hyperlapse from egocentric videos, IEEE Transactions on Circuits and Systems for Video Technology (2017) 11doi:10.1109/tcsvt.2017.2651051

[25] L. Zelnik-Manor, P. Perona, Automating joiners, in: Proceedings of the 5th international symposium on Non-photorealistic animation and rendering - NPAR, ACM Press, 2007. doi: 10.1145/1274871.1274890.

[26] R. Marzotto, A. Fusiello, V. Murino, High resolution video mosaicing with global alignment, in: Proceedings of the 2004 IEEE Computer Society Conference on Computer Vision and Pattern Recognition, 2004. CVPR 2004., IEEE, 2004. doi:10.1109/cvpr.2004.1315099.

[27] P. Khurd, L. Grady, R. Oketokoun, H. Sundar, T. Gajera, S. Gibbs-Strauss, J. V. Frangioni, A. Kamen, Global error minimization in image mosaicing using graph connectivity and its applications in microscopy, Journal of pathology informatics 2 .

[28] G. Golub, C. Van Loan, Matrix Computations, Matrix Computations, Johns Hopkins University Press, 2012. 
[29] F. Lu, E. Milios, Globally consistent range scan alignment for environment mapping, Autonomous robots 4 (4) (1997) 333-349.

[30] S. Agarwal, K. Mierle, Others, Ceres solver, http://ceres-solver.org.

[31] R. Kümmerle, G. Grisetti, H. Strasdat, K. Konolige, W. Burgard, g 2 o: A general framework for graph optimization, in: Robotics and Automation (ICRA), 2011 IEEE International Conference on, IEEE, 2011, pp. 3607-3613.

[32] Y. Hernandez-Mier, W. Blondel, C. Daul, D. Wolf, F. Guillemin, Fast construction of panoramic images for cystoscopic exploration, Computerized Medical Imaging and Graphics 34 (7) (2010) 579-592.

[33] G. Bradski, The OpenCV Library, Dr. Dobb's Journal of Software Tools (2000).

[34] Blender Online Community, Blender - a 3D modelling and rendering package, Blender Foundation, Blender Institute, Amsterdam ( URL http://www . blender .org

[35] A. Goode, B. Gilbert, J. Harkes, D. Jukic, M. Satyanarayanan, OpenSlide: A vendorneutral software foundation for digital pathology, Journal of Pathology Informatics 4 (1) (2013) 27. 